\title{
The Relationship between Thrombolytic Therapy and Hemorrhagic Transformation in Patients with Acute Anterior Circulation Stroke Treated with Mechanical Thrombectomy
}

\author{
Nihat SENGEZE ${ }^{1}$ and Semih GIRAY ${ }^{2 *}$ \\ ${ }^{1}$ Department of Neurology, Suleyman Demirel University Hospital, Turkey \\ ${ }^{2}$ Department of Neurology, Gaziantep University Hospital, Turkey
}

*Corresponding author: Semih GIRAY, Professor, Gaziantep University Hospital, Department of Neurology, Gaziantep, Turkey.

Received Date: December 12, 2019

Published Date: December 18, 2019

\section{Abstract}

Purpose: Hemorrhagic transformation is one of the serious complications that can occur both after mechanical thrombectomy and thrombolytic therapy in acute stroke cases. This study aimed to investigate the relationship between hemorrhagic transformation, thrombolytic therapy and risk factors for stroke in patients with acute anterior circulation large vessel occlusions treated with mechanical thrombectomy.

Methods: The study included a total of 157 patients presented to the hospital with stroke who were found to have anterior circulation occlusion and were treated mechanical thrombectomy. Patients who developed hemorrhagic transformation were divided into groups according to the type of hemorrhage and development of symptomatic hemorrhage.

Results: The mean NIHSS score was $17.9 \pm 4.2$, ASPECTS score $8.4 \pm 1.4$, time from initial symptoms to EVT $4.2 \pm 1.3$ hours. Of the patients who had developed hemorrhage, the hematoma was observed in 46 (59.7\%), petechial hemorrhage was observed in 31 (40.3\%). The rate of hematoma development was significantly higher in patients who underwent IA r-tPA during mechanical thrombectomy compared to the development of petechial hemorrhage ( $\mathrm{p}=0.022)$. Mortality was observed in 64 patients $(40.8 \%)$ at third month.

Conclusion: In conclusion, low ASPECT score, longer time to treatment, and history of diabetes are the reasons that increase the risk of posttreatment hemorrhagic transformation in acute anterior circulation large vessel occlusion cases treated with mechanical thrombectomy. Although there has been no significant increase in the risk of hemorrhagic transformation with IA r-TPA in patients undergoing mechanical thrombectomy, the risk of parenchymal hematoma development has been found to be higher.

Keywords: Mechanical thrombectomy; Anterior circulation stroke; Thrombolytic therapy; Hemorrhagic transformation

Abbreviations: DSA: Digital Subtraction Angiography; mTICI: Modified Thrombolysis in Cerebral Infarction; NIHSS: National Institutes of Health Stroke Scale; EVT: Endovascular Treatment; rtTPA: Tissue-Type Plasminogen Activator; IV: Intravenous; IA: Intra-Arterial

\section{Introduction}

Endovascular therapy has been shown to be effective and safe in acute ischemic stroke (AIS) due to large vessel occlusions. Mechanical thrombectomy is a commonly preferred treatment modality in large vessel occlusions, which is often applied endovascularly by using stent retrievers. In accordance with the principles of acute stroke treatment, intravenous (IV) recombinant tissue-type plasminogen activator (rtPA) thrombolysis should be applied for every eligible patient and this treatment step should never be skipped in patients who are thought to be referred to endovascular treatment (EVT). This recommendation is emphasized as class I and level of evidence A in the latest American Heart Association guideline [1]. Accordingly, IV r-tPA should be started within 4.5 hours for suitable patients and EVT should be performed within six hours after the onset of symptoms [2]. Intra-arterial (IA) r-tPA thrombolysis applied during EVT has been also proven to 
be also effective and safe [3]. Hemorrhagic transformation is one of the serious complication that can occur both after mechanical thrombectomy and thrombolytic therapy in acute stroke cases. Furthermore, it increases the risk of functional poor outcome and mortality $[3,4]$.

Hemorrhagic transformations developing inside the infarct tissue after stroke treatment are classified as petechial hemorrhages with no mass effect in the tissue and hematomas with a space-occupying effect [5]. Therefore, it is of great importance to determine the risk factors that may predict the development of hemorrhagic transformation in patients to be treated with mechanical thrombectomy and to determine the use of thrombolytic therapy in these risky patients and a framework for treatment dose to be used. This study aimed to investigate the relationship between hemorrhagic transformation, thrombolytic therapy and risk factors for stroke in patients with acute anterior circulation large vessel occlusions treated with mechanical thrombectomy.

\section{Material and Methods}

The study included a total of 157 patients presented to the hospital with stroke between January 2015 and March 2019, who were found to have anterior circulation occlusion (Middle cerebral artery [MCA] M1, internal carotid artery [ICA], and tandem occlusions) and were treated with endovascular therapy using mechanical thrombectomy. Studyinclusion criteria were determined to be as follows: being admitted to the emergency department of Gaziantep University due to stroke and receiving the acute stroke diagnosis, being treated with mechanical thrombectomy using the stent retriever, undergoing EVT within the first six hours, and not having any other hematologic disease that may cause hemorrhage. Seven patients who were treated with IA r-TPA and did not undergo mechanical thrombectomy were excluded from the study. IA r-TPA was administered up to a maximum of $20 \mathrm{mg}$.

Hemogram, routine biochemical examination (fasting blood glucose, urea, creatinine, blood urea nitrogen), sedimentation value, prothrombin time (PT), activated partial thromboplastin time (aPTT), and international normalized ratio (INR) of all cases included in the study were examined. Patients who developed hemorrhagic transformation at the end of the study were divided into groups according to the type of hemorrhage and development of symptomatic hemorrhage. The evaluation of hemorrhagic transformation at CT was performed by two different neurologists blind to patients outcomes. Hounsfield units (hu) values on CT were used to decide on hemorrhage and contrast discrimination. The patients were classified and evaluated according to Heidelberg and European Cooperative Acute Stroke Study III (ECASS III) classifications for hemorrhagic transformation. Symptomatic hemorrhage was defined as an increase of $\geq 4$ in the National Institutes of Heart Stroke Scale (NIHSS) score and clinical deterioration after hemorrhage [6-8]. Patients were evaluated for functional outcome and mortality using the modified Rankin Scale (mRS) at the end of the third month. This study was approved by the Gaziantep University Clinical Research Ethics Committee with the decision no 2019/163.

\section{Statistical analysis}

Statistical analysis was performed using SPSS (Statistical Package for Social Sciences) version 23. Non-normally distributed data were compared using non-parametric tests and normally distributed data were compared using parametric tests. Chi-square test was used to compare the grouped data. Data were expressed as mean \pm standard deviation (SD) (normally distributed data, mean-range, non-normally distributed data) and percentage (\%). The relationship between continuous variables was analyzed by Pearson's correlation coefficient and Spearman's rank correlation coefficient was used for the non-parametric variables. Results were evaluated at a $95 \%$ confidence interval and a p value of $<0.05$ was considered statistically significant.

\section{Results}

Of the 157 patients included in the study, 55.4\% (n=87) were women. The mean age was $62.9 \pm 12.9$ years. Right-sided stroke was observed in 78 (49.7\%) of the patients. The mean NIHSS score at the time of admission was $17.9 \pm 4.2$, the mean Alberta Stroke Program Early Computed Tomography (CT) Score (ASPECTS) score was $8.4 \pm 1.4$, the mean time from initial symptoms to EVT was $4.2 \pm 1.3$ hours, and the total number of thrombectomy pass performed was $2.2 \pm 0.8$. Of the patients undergoing thrombectomy, 89 (56.7\%) had MCA occlusion, 21 (13.4\%) had ICA peak occlusion, and 47 (29.9\%) had tandem occlusion.

Twenty-eight $(17.8 \%)$ patients were treated with IV r-TPA whereas 101 (64.3\%) patients received IA r-TPA. Mortality was observed in 64 patients (40.8\%) at the end of the third month.

Control CT of the brain performed following the treatment showed hemorrhage in 77 (49\%) of patients. Of the patients who had developed hemorrhage, the hematoma was observed in $46(59.7 \%)$ whereas petechial hemorrhage was observed in 31 patients $(40.3 \%)$. Forty-one patients with hemorrhage had clinical deterioration causing an increase of 4 points and more in the NIHSS score. Demographic data were compared in terms of hemorrhage development after mechanical thrombectomy, risk factors for stroke, and treatment used in all patients included in the study (Table 1).

The hemorrhage rate was significantly higher in patients with diabetes than those without diabetes $(p=0.008)$. Third-month mortality rate was significantly higher in patients with hemorrhagic transformation after mechanical thrombectomy ( $\mathrm{p}=0.032)$.

When the patients were compared in terms of clinical evaluation scores, laboratory and imaging data and hemorrhage rates after mechanical thrombectomy (Table 2), the development of hemorrhagic transformation was significantly higher in patients whose pre-treatment ASPECT scores were low and who were taken into the EVT at a later time ( $\mathrm{p}=0.003$ and $\mathrm{p}=0.001$, respectively) (Table 2).

The third-month poor functional outcome (mRS) was significantly higher in patients with hemorrhagic transformation after mechanical thrombectomy $(p=0.014)$. Seventy-seven patients 
with hemorrhagic transformation after mechanical thrombectomy were divided into two groups according to the type of hemorrhage: petechial hemorrhage and hematoma (Table 3). The rate of hematoma development was significantly higher in patients who underwent IA r-tPA during mechanical thrombectomy compared to the development of petechial hemorrhage $(p=0.022)$. There was no statistically significant difference between the two types of hemorrhage in terms of other risk factors (age, ASPECT score, diabetes, atrial fibrillation, etc.) for hemorrhagic transformation. However, the rate of symptomatic hemorrhage, third-month poor clinical outcomes ( $m R S \geq 3$ ), and third-month mortality rates were significantly higher in patients with hematoma $(p=0.001, p=0.001$, and $\mathrm{p}=0.009$, respectively) (Table 3 ).

Table 1: Comparison of demographic data in terms of stroke risk factors and hemorrhagic transformation after mechanical thrombectomy.

\begin{tabular}{|c|c|c|c|}
\hline \multirow{2}{*}{ Stroke risk factors and patients data } & \multicolumn{2}{|c|}{ Hemorrhagic transformation after mechanical thrombectomy } \\
\cline { 2 - 4 } & No hemorrhage & hemorrhage & p* \\
\cline { 2 - 4 } & $\mathrm{n}(\%)$ & $\mathrm{n}(\%)$ & 0.432 \\
\hline Hypertension & $46(\% 48.4)$ & $49(\% 51.6)$ & 0.998 \\
\hline Atrial fibrillation & $27(\% 50.9)$ & $26(\% 49.1)$ & 0.498 \\
\hline Smoke & $24(\% 47.1)$ & $27(\% 52.9)$ & $0.008^{*}$ \\
\hline Diabetes & $25(\% 38.5)$ & $40(\% 61.5)$ & 0.805 \\
\hline CABG history & $6(\% 54.5)$ & $5(\% 45.5)$ & 0.248 \\
\hline Coroner artery disease & $23(\% 59)$ & $16(\% 41)$ & 0.171 \\
\hline Previous stroke & $3(\% 30)$ & $7(\% 70)$ & 0.722 \\
\hline Hyperlipidemia & $26(\% 53.1)$ & $23(\% 46.9)$ & 0.76 \\
\hline IV r-TPA & $15(\% 53.6)$ & $13(\% 46.4)$ & 0.858 \\
\hline IA r-TPA & $52(\% 51.5)$ & $49(\% 48.5)$ & 0.108 \\
\hline Varfarin medication & $10(\% 71.4)$ & $4(\% 28.6)$ & 0.187 \\
\hline NOAC's medication & $4(\% 80)$ & $1 \% 20)$ & 0.068 \\
\hline Antiaggregant medication & $14(\% 37.8)$ & $23(\% 62.2)$ & 0.303 \\
\hline First pass recanalization $<45$ min & $14(\% 60.9)$ & $9(\% 39.1)$ & $0.032^{*}$ \\
\hline Mortality at third month & $26(\% 40.6)$ & $38(\% 59.4)$ & 0.911 \\
\hline Succesful recanalization $(m T I C I \geq 2 b)$ & $66(\% 51.2)$ & & \\
\hline
\end{tabular}

${ }^{*}$ Chi-square test,

CABG: Coronary artery bypass surgery, NOAC's: New oral anticoagulants, mTICl: modified treatment in cerebral infarction, IV r-TPA: Intravenous recombinant tissue plasminogen activator IA r-TPA: Intra-arterial recombinant tissue plasminogen activator.

Table 2: Comparison of laboratory, imaging and clinical findings on admission of stroke and hemorrhagic transformation after mechanical thrombectomy.

\begin{tabular}{|c|c|c|c|}
\hline \multirow{2}{*}{$\begin{array}{c}\text { Laboratory, imaging and clinical } \\
\text { findings on admission }\end{array}$} & \multicolumn{2}{|c|}{ Hemorrhagic transformation after mechanical thrombectomy } \\
\cline { 2 - 4 } & No hemorrhage & hemorrhage & Median [25-75] \\
\cline { 2 - 4 } & $63[51-71]$ & $68[57-74]$ & 0.077 \\
\hline Age & $9[8-10]$ & $8[7-9]$ & $0.003^{*}$ \\
\hline Aspect score & $17[16-20]$ & $19[16-21]$ & 0.059 \\
\hline NIHSS on admission & $4[3-4]$ & $5[4-6]$ & $0.001^{*}$ \\
\hline Time from stroke onset to groin \\
puncture (hours) & $2[2-3]$ & $2[2-3]$ & 0.819 \\
\hline Total number of passes & $144[113-190]$ & $152[121-201]$ & 0.3 \\
\hline Glucose on admission & $9890[8270-12850]$ & $10370[8470-12770]$ & 0.523 \\
\hline Leukocyte & $240[201-302]$ & $263[210-312]$ & 0.234 \\
\hline Platelet & $0.78[0.62-1.0]$ & $0.78[0.6-0.9]$ & 0.461 \\
\hline Creatinin & $14.2[13.6-15.5]$ & $14.3[13.5-15.3]$ & 0.971 \\
\hline RDW & $3[2-6]$ & $5[3-6]$ & $0.014^{*}$ \\
\hline mRS at third month & & & \\
\hline
\end{tabular}

**Mann-whitney U test

RDW: Red Cell Distribution Width, NIHSS: National Institues Of Healt Stroke Scale, mRS: modified Rankin Scale 
Table 3: Comparison of Baseline characteristics of patients and hemorrhagic transformation type after mechanical thrombectomy.

\begin{tabular}{|c|c|c|c|}
\hline \multirow{3}{*}{ Baseline characteristics } & \multicolumn{3}{|c|}{ Hemorrhagic transformation after mechanical thrombectomy } \\
\hline & Petechial Hemorrhage & Parenchymal Hematoma & \multirow{2}{*}{$\mathbf{p}^{*}$} \\
\hline & n (\%) & n (\%) & \\
\hline Sex (Female) & $16(35.6)$ & $39(64.4)$ & 0.318 \\
\hline Atrial fibrillation & $9(34.6)$ & $17(65.4)$ & 0.471 \\
\hline Hypertension & $21(42.9)$ & $28(57.1)$ & 0.539 \\
\hline Smoke & $10(37)$ & $17(63)$ & 0.672 \\
\hline Diabetes & $15(37.5)$ & $25(62.5)$ & 0.608 \\
\hline Coroner artery disease & $8(50)$ & $8(50)$ & 0.372 \\
\hline Previous stroke & $2(28.6)$ & $5(71.4)$ & 0.508 \\
\hline Hyperlipidemia & $10(43.5)$ & $13(56.5)$ & 0.707 \\
\hline IA r-TPA & $15(30.6)$ & $34(69.4)$ & $0.022^{*}$ \\
\hline IV r-TPA & $5(38.5)$ & $8(61.5)$ & 0.885 \\
\hline Antiaggregant medication & $7(30.4)$ & $16(69.6)$ & 0.251 \\
\hline Succesful recanalization (mTICI $\geq 2 \mathrm{~b}$ ) & $26(41.3)$ & 37 (58.7) & 0.701 \\
\hline First pass recanalization $<45 \mathrm{~min}$ & $4(44.4)$ & $5(55.6)$ & 0.785 \\
\hline Symptomatic hemorraghea & $5(12.2)$ & $36(87.8)$ & $0.001^{*}$ \\
\hline Mortality at third month & $7(18.4)$ & $31(81.6)$ & $0.001^{*}$ \\
\hline \multirow[t]{2}{*}{ Good functional outcome at third month (mRS $\leq 2)$} & $10(71.4)$ & $4(28.6)$ & $0.009^{*}$ \\
\hline & Median [25-75] & Median [25-75] & $\mathbf{p}^{* *}$ \\
\hline Age & $64[56-74]$ & $68[58-78]$ & 0.470 \\
\hline Aspect score & $8[7-10]$ & 8 [7-9] & 0.869 \\
\hline NIHSS on admission & $16[14-21]$ & 19 [18-21] & 0.278 \\
\hline Total number of passes & $2[2-3]$ & $2[2-3]$ & 0.978 \\
\hline Glucose & 137 [119-181] & 156 [122-209] & 0.568 \\
\hline Leukocyte & $10370[8600-13330]$ & $10315[8400-12770]$ & 0.959 \\
\hline Platelet & 258 [209-344] & 265 [210-308] & 0.689 \\
\hline Hemoglobin & $14[12-14.6]$ & $12.7[11.4-14]$ & 0.152 \\
\hline RDW & $14.1[13.2-15.5]$ & $14.3[13.6-15.1]$ & 0.564 \\
\hline Creatinin & $0.7[0.6-0.9]$ & $0.8[0.6-0.9]$ & 0.450 \\
\hline
\end{tabular}

${ }^{*}$ Chi-square test, **Mann-whitney $\mathrm{U}$ test

IV r-TPA: Intravenous recombinant tissue plasminogen activator, IA r-TPA: Intra-arterial recombinant tissue plasminogen activator, mTICI: modified treatment in cerebral infarction, mRS: modified Rankin Scale, RDW: Red Cell Distribution Width, NIHSS: National Institues Of Healt Stroke Scale

\section{Discussion}

The present study has demonstrated that the development of hemorrhagic transformation after EVT is an independent risk factor for both third-month mortality and poor outcome. In similar studies, development of parenchymal hematoma has been reported to be associated with poor functional outcome and mortality $[9,10]$. Pre-treatment low ASPECT scores, longer time to treatment and the presence of a history of diabetes before the stroke are the factors that increase the risk of hemorrhagic transformation after mechanical thrombectomy. In the literature, factors that increase the risk of hemorrhagic transformation after mechanical thrombectomy have been reported to be high NIHSS score at the time of admission, history of diabetes, atrial fibrillation, proximal vessel occlusion, time to endovascular treatment, high serum glucose, wake-up stroke and high systolic blood pressure $[4,5,9]$. In a study by Mokin M. et al. [3] involving patients with acute stroke treated with IA and IV r-tPa, low ASPECT score $(<8)$, hyperglycemia and high blood pressure have been reported to be important risk factors for the development of hemorrhagic transformation.

In the present study, Forty-one patients with hemorrhage had clinical deterioration causing an increase of 4 points and more in the NIHSS score. Even, thirty-six (88\%) of these patients also developed parenchymal hematoma. The development of hemorrhagic transformation was found to be higher in cases where the time from onset of stroke symptoms to treatment was long. We think that the long period of time until the patients (especially from remote regions) in endovascular therapy were processed contributed to this result. In a review by Sussman ES et al. [8] where the data of the studies on IV r-tPA treatment in the acute anterior circulation stroke cases were collected, the post-treatment hemorrhagic transformation rates were reported to be $39.6 \%$ and $27 \%$ for asymptomatic hemorrhagic transformation and $8.8 \%$ and $2.4 \%$ for symptomatic hemorrhagic transformation in the ECASS II and III studies, respectively. Similarly, post-treatment hemorrhagic 
transformation rates were reported to be $50 \%$ and $68 \%$ for asymptomatic transformation and $15.4 \%$ and $10 \%$ for symptomatic transformation in PROACT I and II studies on IA r-tPA application, respectively. The common aspect of studies reporting high rates of hemorrhagic transformation was that the time from onset of stroke symptoms to treatment was 5.5 and 4.5 hours, respectively. Asymptomatic hemorrhagic transformation rates in patients with anterior circulation stroke treated with mechanical thrombectomy have been reported to be $42.9 \%$ in Mechanical Embolus Removal in Cerebral Ischemia (MERCI) trial, 30\% in SOLITARE trial, and 40.9\% in the TREVO study [8]. In the analysis of these studies, it has been reported that the combination of IV and IA thrombolytic therapy could not be the main cause of high hemorrhagic transformation rates, but the inclusion criteria of the studies have been reported to be effective in the high hemorrhagic transformation rates.

In the present study, there was no significant increase of risk in terms of successful recanalization (TICI $\geq 2 \mathrm{~b}$ ) and hemorrhage development due to higher number of recanalization maneuvers with stent retrievers. Neuberger U. et al. (4) investigated the hemorrhage risk factors after mechanical thrombectomy in anterior circulation occlusions and reported that patients with infarct size $>1 / 3$ of vascular territory, higher serum glucose levels, higher thrombectomy maneuver count, and right hemisphere occlusions were significantly associated with a higher risk of developing hemorrhage. In the same study, wake-up strokes, higher serum glucose levels and being transferred from an external clinic were also revealed as risk factors for development of symptomatic hemorrhage. Furthermore, complete recanalization of TICI 3 was determined to be a factor that reduced the risk of developing hemorrhage. Compatible with the present study, the use of IV r-tPA, oral anticoagulants and antiplatelets during mechanical thrombectomy have been found to be factors that do not pose a risk for the development of hemorrhagic transformation [4].

In the present study, the history of hyperlipidemia was more frequent in patients without hemorrhagic transformation but no statistically significant result was obtained in this regard. Lee YB et al. [5] found in their study that successful recanalization and hyperlipidemia were factors that reduce the risk of hemorrhagic transformation. The authors further reported that the chronic inflammatory process in the vascular wall caused by hyperlipidemia could be a possible factor reducing the risk of hemorrhage after recanalization. In this study, IV r-TPA, IA r-TPA, the use of oral anticoagulants or antiplatelets before the stroke have been found to not increase the risk of hemorrhagic transformation. When the patients who developed hemorrhagic transformation after mechanical thrombectomy were examined in two groups according to the type of hemorrhage (petechial hemorrhage and hematoma), IV r-TPA, the use of oral anticoagulants and antiaggregants before the stroke development were found to not cause an increase in the risk of parenchymal hematoma. However, in cases where hemorrhagic transformation occurred in patients treated with IA r-tPA, the risk of this hemorrhage being in the form of parenchymal hemorrhage was found to be statistically significant $(p=0.022 *)$.
There was no statistically significant difference between patients with petechial hemorrhage and those with hematoma in terms of other risk factors, even including diabetes.

The data has potential interesting aspects, as the role of additional IA rtPA in contemporary MT still needs to be defined. Therefore, an increased risk of sICH / PH after IA rtPA administration is of interest. In a study by Nogueira RG et al. [9], the use of IV r-TPA in anterior circulation occlusions treated with EVT has been found to increase the risk of hemorrhagic transformation after the treatment. However, in the same study, an inverse relationship has been found between the use of IA r-TPA and the risk of hematoma and it has been reported that this inverse relationship may be attributed to the selection bias, where patients with a more favorable imaging profile would have preferably received IA r-tPA. The inclusion of patients without large vessel occlusion may have affected this finding [9]. Similarly, in a study by Kaesmacher J. et al. [10], IV r-TPA and oral anticoagulants have been found to not increase the risk of hemorrhagic transformation after mechanical thrombectomy. In a study by Christoforidis G.A. et al. [11,12], poor pial collateral formation documented on angiography in patients with both anterior and posterior circulation occlusion has been found to be a higher risk factor for the hemorrhagic transformation after IA thrombolytic therapy.

\section{Conclusion}

In conclusion, low ASPECT score, longer time to treatment, and history of diabetes are the reasons that increase the risk of post-treatment hemorrhagic transformation in acute anterior circulation large vessel occlusion cases treated with mechanical thrombectomy. Although there has been no significant increase in the risk of hemorrhagic transformation with IA r-TPA in patients undergoing mechanical thrombectomy, the risk of parenchymal hematoma development has been found to be higher. Considering the findings of our study, we believe that it is of great importance to carry out further studies to determine the IA r-tPA application in patients at high risk of developing hemorrhagic transformation after mechanical thrombectomy and to determine the dose of treatment that can be administered.

\section{Acknowledgement}

None.

\section{Conflict of Interest}

No conflict of interest.

\section{References}

1. Bektas H, Çabalar M, Topcuoglu MA, Arsava EM (2016) Endovascular Treatment in Acute Ischemic Stroke: What Has Changed Over the Last One Year? Turkish journal of cerebrovascular diseases 22(1): 1-8.

2. Dinc Y, Uzuner GT, Emir B (2018) Evaluation of Seizure After Stroke in Stroke Unit. Turk J Neurol 24(2): 153-158

3. Mokin M, Kan P, Kass-Hout T, Abla AA, Dumont TM, et al. (2012) Intracerebral hemorrhage secondary to intravenous and endovascular intraarterial revascularization therapies in acute ischemic stroke: an update on risk factors, predictors, and management. Neurosurg Focus 32(4): E2. 
4. Neuberger U, Kickingereder P, Schönenberger S, Schieber S, Ringleb PA, et al. (2019) Risk factors of intracranial hemorrhage after mechanical thrombectomy of anterior circulation ischemic stroke. Neuroradiology 61(4): 461-469.

5. Lee YB, Yoon W, Lee YY, Kim SK, Baek BH, et al. (2019) Predictors and impact of hemorrhagic transformations after endovascular thrombectomy in patients with acute large vessel occlusions. J Neurointerv Surg 11(5): 469-473.

6. Hacke W1, Donnan G, Fieschi C, Kaste M, von Kummer R, et al. (2004) Association of outcome with early stroke treatment: pooled analysis of ATLANTIS, ECASS and NINDS rt-PA stroke trials. Lancet 363: 768-774.

7. Bluhmki E, Chamorro A, Dávalos A, Machnig T, Sauce C, et al. (2009) Stroke treatment with alteplase given 3.0-4.5 h after onset of acute ischaemic stroke (ECASS III): additional outcomes and subgroup analysis of a randomised controlled trial. Lancet Neurol 8(12): 1095-1102.

8. Sussman ES, Connolly ES (2013) Hemorrhagic transformation: a review of the rate of hemorrhage in the major clinical trials of acute ischemic stroke. Front Neurol 4: 69.
9. Nogueira RG, Gupta R, Jovin TG, Levy EI, Liebeskind DS, et al. (2015) Predictors and clinical relevance of hemorrhagic transformation after endovascular therapy for anterior circulation large vessel occlusion strokes: a multicenter retrospective analysis of 1122 patients. J Neurointerv Surg 7: 16-21.

10. Kaesmacher J, Kaesmacher M, Maegerlein C, Zimmer C, Gersing AS, et al. (2017) Hemorrhagic transformations after thrombectomy: risk factors and clinical relevance. Cerebrovasc Dis 43: 294-304.

11. Christoforidis GA, Karakasis C, Mohammad Y, Caragine LP, Yang M, et al. (2009) Predictors of hemorrhage following intra-arterial thrombolysis for acute ischemic stroke: the role of pial collateral formation. AJNR Am J Neuroradiol 30(1): 165-170.

12. Bang OY, Saver JL, Kim SJ, Kim GM, Chung CS, et al. (2011) UCLA-Samsung Stroke Collaborators. Collateral flow averts hemorrhagic transformation after endovascular therapy for acute ischemic stroke. Stroke 42(8): 2235-2239. 